\title{
A TEMPERATURA E O DESENVOLVIMENTO LARVAL DE CHIRONOMUS SANCTICAROLI (DIPTERA: CHIRONOMIDAE)
}

GIOVANNI STRIXINO

SUSANA TRIVINHO STRIXINO

\begin{abstract}
Development rate, survival and growth of a neotropical midge, Chironornus sancticaroli, were determined in laboratory at different temperatures. The "lower thermal limit" is $12^{\circ} \mathrm{C}$ and the "thermal constant", characteristic for the species, varies from 140-144 day-degree. These results pointed out to a high development when compared with species of temperate areas and permit to expect, for $\mathrm{C}$. sancticaroli, 16-18 generations/year, in natural conditions.
\end{abstract}

A maioria das espécies de Chironomidae completa o seu desenvolvimento em torno de temperaturas que variam de $0^{\circ} \mathrm{C}$ a $32^{\circ} \mathrm{C}$ (Curry, 1965, in Oliver, 1971). O aumento da temperatura acelera não só as reações químicas, como os processos fisiológicos em geral, notadamente o desenvolvimento e o crescimento. Conseqüentemente, a duração de uma geração e o número de gerações/ano varia com a latitude ou o seu equilavente ecológico (Oliver, op. cit.). Estudos com espécies de regiões tropicais têm mostrado que estas, encontrando condições térmicas favoráveis, podem completar seu ciclo em menos de 15 dias (Syrjämäki, 1965, Dejoux, 1971; Strixino \& Strixino, 1982b), o que faz prever um elevado número de gerações por ano.

O presente trabalho propõe-se fundamentalmente a fornecer informações sobre a taxa de desenvolvimento de uma espécie regional, Chiroromus sancticaroli, mantida a diferentes temperaturas em condições de laboratório. Secundariamente, através da Somação Térmica (graus-dia), pretendeu-se estimar, numa fase preliminar, o número de gerações prováveis no ambiente natural.

Tais conhecimentos, básicos e imprescindíveis para estimativas da produção de biomassa (Winberg, 1971), são raros em regiões tropicais, restringindo-se a maior parte das informações a espécies de Chironomidae que vivem em regiões temperadas ou árticas (Konstantinov, 1958; Mackey, 1977; Butler et al., 1980).

\section{MATERIAL E METODOS}

Massas ovígeras de $C$. sancticaroli, obtidas a partir de fêmeas capturadas nas adjacências da Represa do Monjolinho (Campus da UFSCar), foram depositadas em placas de Petri contendo água, e mantidas até que as larvas do I ínstar tivessem eclodido e abandonado a mucilagem gelatinosa. Estas foram, em seguida, transferidas para bandejas de aço-inox contendo água (aproximadamente 4 litros) e supridas com Avemicina-Purina (0,4 g/100 larvas/semana).

Duas séries de bandejas, assim preparadas, foram cobertas por gaiolas de nylon para a captura dos adultos e mergulhadas em banhos de temperatura constante $\left(12^{\circ} \mathrm{C}, 15^{\circ} \mathrm{C}, 20^{\circ} \mathrm{C}, 22^{\circ} \mathrm{C}, 25^{\circ} \mathrm{C}, 30^{\circ} \mathrm{C}, 35^{\circ} \mathrm{C}\right)$ em dispositivo descrito por Rantin (1978).

Durante o experimento foram anotadas a duração média do desenvolvimento e a sobrevivência em cada temperatura. O peso das fêmeas emergidas foi estimado através de técnica desenvolvida por Strixino (1980).

\section{RESULTADOS E DISCUSSÃO}

A Fig 1a mostra a duração da fase larval (D), em dias, a diferentes temperaturas. O fenômeno curvilíneo mantém uma relação inversa com a tempe- 


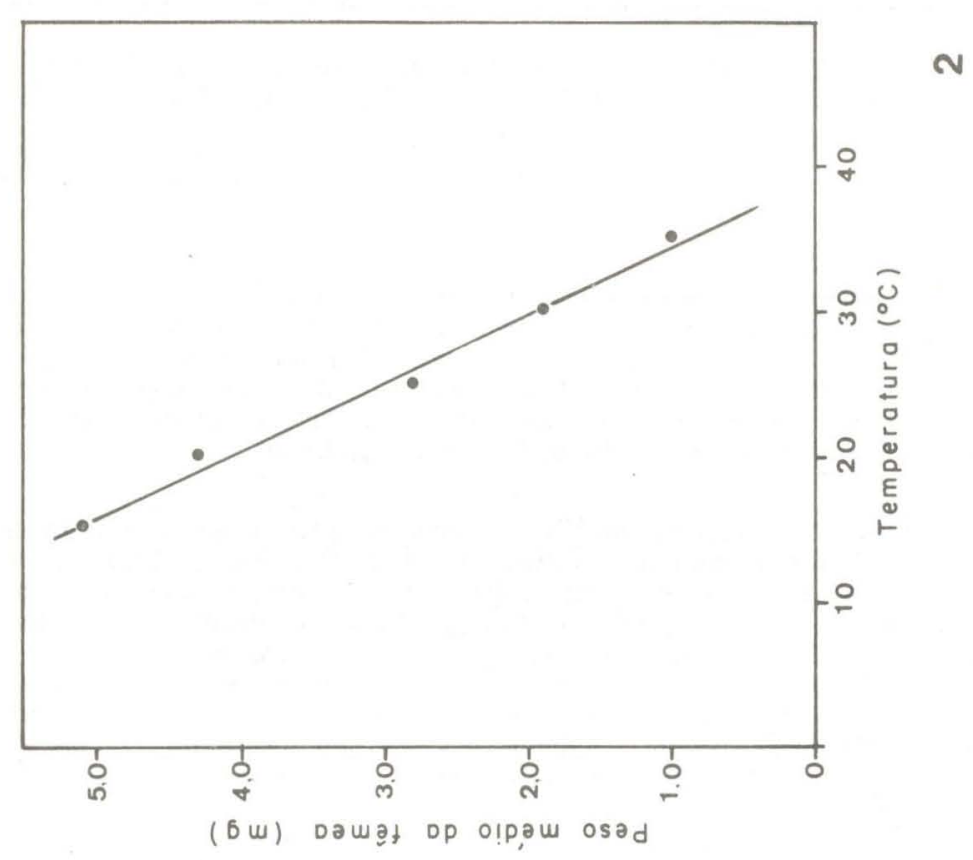

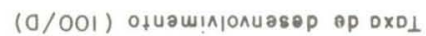

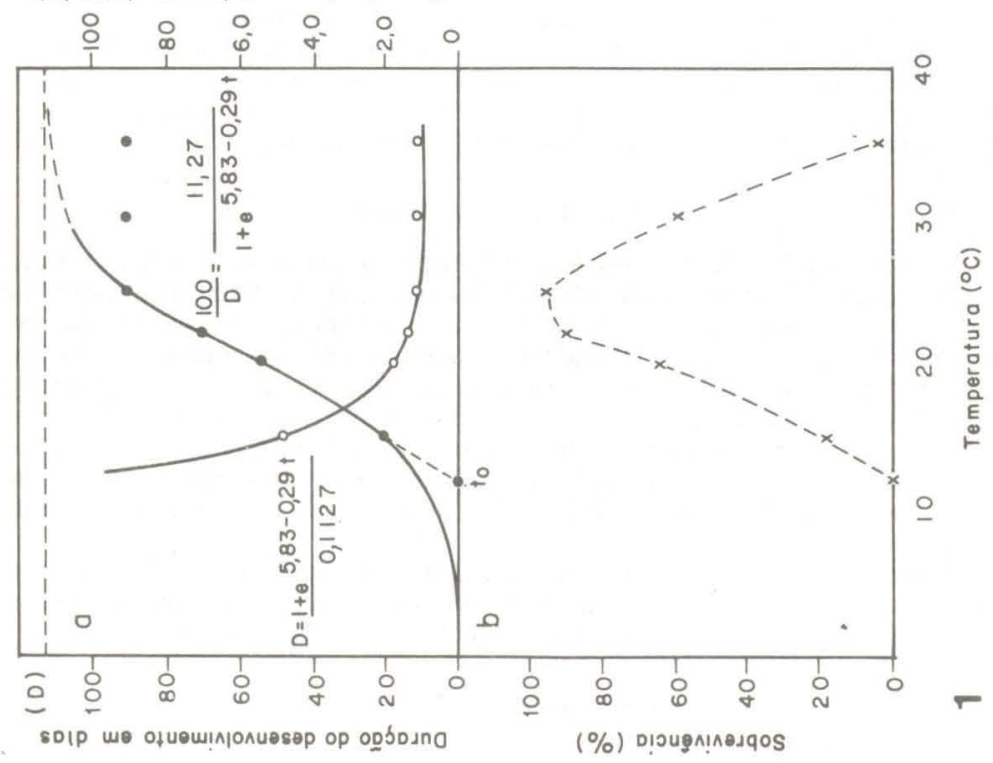


ratura; a $20^{\circ} \mathrm{C}$ a duração do desenvolvimento é aproximadamente o dobro da duração a $25^{\circ} \mathrm{C}$. As temperaturas mais favoráveis encontram-se compreendidas no intervalo de $18^{\circ} \mathrm{C}$ e $31^{\circ} \mathrm{C}$, registrando-se entre $22^{\circ} \mathrm{C}-26^{\circ} \mathrm{C}$ as maiores sobrevivências (Fig 1b).

Os dados apresentados na Fig. 1a mostram claramente a dependência da velocidade de desenvolvimento (1/D) das larvas de $C$. sancticaroli com a temperatura. A relação entre taxa de desenvolvimento e temperatura é do tipo sigmóide e a expressão logística representa adequadamente o fenômeno (Davidson, 1944; Browning, 1952). O ponto de inflexão da curva ocorre a $20^{\circ} \mathrm{C}$. A temperatura crítica $\left(\mathrm{t}_{0}\right)$, calculada pela intersecção do intervalo de linearidade da curva com a abcissa, aponta valor próximo de $12^{\circ} \mathrm{C}$, idêntico ao obtido experimentalmente, quando cessa o desenvolvimento. $\mathrm{O}$ valor bastante elevado de $t_{0}$, comparado com outros obtidos para o gênero por Konstantinov (1958) e Mackey (1977), sugere uma característica adaptativa da espécie neotropical às condições térmicas do ambiente no qual normalmente vive.

$\mathrm{O}$ peso médio da fêmea ao emergir decresce linearmente com o número da temperatura (Fig. 2). O desenvolvimento é mais acelerado do que o crescimento, com a elevação da temperatura (Mackey, op. cit.), resultando em adultos menores.

Dentro do intervalo de linearidade da curva da taxa de desenvolvimento, é possível estabelecer a relação temperatura-duração pela Regra da Somação Térmica (Wigglesworth, 1972). A constante térmica obtida, característica de C. sancticaroli, é temperatura-independente e está compreendida entre 140-144 graus-dias, valor este muito baixo quando comparado com aqueles registrados por Konstantinov (op. cit.) para C. dorsalis (308-384 graus-dia), C. annularius (360-395 graus-dia), C. plumosus (595-640 graus-dia) e C. heterodentatus (300-342 graus-dia).

Tomando por base as condições térmicas normalmente encontradas na re-

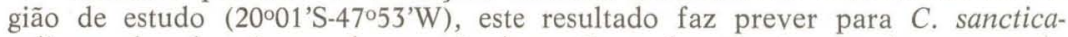
roli um elevado número de gerações/ano. O quadro térmico geral apresentado pela Represa do Monjolinho (Strixino \& Strixino, 1982a) indica a existência de dois períodos bem marcados: época fria (maio-outubro), com temperaturas médias mensais da água variando de 15,0 a $19,5^{\circ} \mathrm{C}$; época quente (novembroabril) com temperaturas entre $21,0-24,0^{\circ} \mathrm{C}$. Na primeira época, a duração aproximada do desenvolvimento larvar seria de 28-30 dias e na segunda, de 15-17 dias. Anualmente, teríamos portanto a possibilidade de 16-18 gerações, indicando a potencialidade para o desenvolvimento ininterrupto da espécie neste ambiente.

\section{REFERENCIAS}

Browning, T. O., 1952. The influence of temperature on the rate of development of Insects, with special reference to the eggs of Gryllulus commodus Walker. Aust. J. Scient. Res. Ser. B, 5(1): 96-111.

Butler, M., M. C. Miller \& S. Mozley (1980). Macrobentos. In: Limnology of Tundra Ponds. Hobbie, J. E. (ed.) V. S./IBP synthesis series 13. Dowden, Hutchinson \& Ross, Inc. Stroudsburg 514 pp: 297-3.39.

Davidson, J., 1944. On the relationship between temperature and the rate of development of insects at constant temperatures. J. Anim. Ecol., 13: 26-38.

Dejoux, C. 1971. Recherches sur le cycle de développement de Chironomus pulcher (Diptera: Chironomidae). Can. Ent. 103: 465-470.

Konstantinov, A. S., 1958. "A influência da temperatura na velocidade de crescimento e de desenvolvimento das larvas de Chironomidae". Dokl. Akad Nauk. S. S. S. R. 120 (6): 1362-1365 (em Russo).

Mackey, A. P., 1971. Growth and development of larval Chironomidae. Oikos, 28 (2-3): 270-275.

Oliver, D. R., 1971. Life history of the Chironomidae. Ann. Rev. Entomol., $16: 221-230$.

Rantin, F. T., 1978. Temperaturas letais, aclimatação e tolerância térmica do acará G. brasiliensis. (Quoy \& Gaimard, 1824) - Represa do Broa, Represa 
da UFSCar Estado de São Paulo. Dissertação de Mestrado Inst. Biociências, USP. $181 \mathrm{p}$.

Strixino, S. T., 1980. Estudos sobre a fecundidade de Chironomus sancticaroli sp. n. (Diptera: Chironomidae). Tese de Doutorado Inst. Biociências, USP. $157 \mathrm{p}$

Strixino, G. \& Strixino, S. T., 1982a. Macrobentos da represa do Monjolinho (São Carlos, SP). Revta bras. Biol. 2(1): 165-179.

Strixino, S. T. \& Strixino, G., 1982b. Ciclo de vida de Chironomus sancticaroli (Diptera: Chironomidae). Revta bras. Ent., 26(2): 183-189.

Syrjämäki, J. 1965. Laboratory studies on the swarming behaviour of Chironomus strenzkei Fittkau in Litt. (Dipt., Chironomidae). I. Mechanism of swarming and mating. Ann. Zool. Fenn. 2: 145-152.

Wigglesworth, V. B., 1972. The Principles of Insect Physiology. London, Chapman and Hall. 827 pp.

Winberg, G. G. (ed.), 1971. Methods for the Estimation of Production of Aquatic Animals. Academic Press Inc. (London) Ltd, London and New York. $175 \mathrm{p}$. 